\title{
Dynamic Analysis of Serum MMP-7 and Its Relationship with Disease Progression in Biliary Atresia: A Multicenter Prospective Study
}

\section{Shuiqing Chi}

Wuhan Union Hospital

\section{Peipei Xu}

Wuhan Union Hospital

Pu Yu

Xi'an children's hospital

\section{Guoqing Cao}

Wuhan Union Hospital

\section{Haibin Wang}

Wuhan Childrens Hospital: Wuhan Women and Children Medical Care Center

\section{Yongqin Ye}

Shenzhen Children's Hospital

\section{Shuai Li}

Wuhan Union Hospital

Yun Zhou

Wuhan Union Hospital

Xiangyang Li

Wuhan Union Hospital

Ying Zhou

Wuhan Union Hospital

\section{Xi Zhang}

Wuhan Union Hospital

Huizhong Niu

Children's Hospital of Hebei

\section{Lei $\mathrm{Xu}$}

Wuhan Union Hospital

\section{Pengcheng Cai}

Wuhan Union Hospital

Shaotao Tang ( $\nabla$ tshaota083@126.com )

Huazhong University of Science and Technology 


\section{Research Article}

Keywords: Biliary atresia, cholestatic liver diseases, Diagnosis, Prognosis, MMP7, Kasai portoenterostomy, Neonates, diagnostic biomarker, dynamic monitoring, Native liver survival.

Posted Date: September 27th, 2021

DOI: https://doi.org/10.21203/rs.3.rs-866431/v1

License: (c) (i) This work is licensed under a Creative Commons Attribution 4.0 International License. Read Full License 


\section{Abstract}

\section{Purpose}

We aimed to assess the dynamic changing trend of serum matrix metalloproteinase-7 (MMP-7) in BA patients from diagnosis to LTx to further elucidate its clinical value in diagnosis and prognoses and its relationship with disease progression.

\section{Methods}

In this multicentre prospective study, a total of 440 cholestasis patients (direct bilirubin level of $>17$ $\mu \mathrm{mol} / \mathrm{L}$ ) were enrolled. Serum and stool MMP-7 levels were measured using an enzyme-linked immunosorbent assay at different time points and analysed. The polymorphism of MMP-7 was assessed to explore the possible reasons for the low value in BA patients.

\section{Results:}

In neonate biliary atresia patients, using a cut-off value of $>26.73 \mathrm{ng} / \mathrm{ml}$, serum MMP-7 had a AUC of 0.954. A genetic mutation (G137D) and rapid degradation of MMP-7 in serum samples could cause low MMP-7 levels in serum of BA patients. Four dynamic patterns of serum MMP-7 post-KPE were associated with prognosis. A high concentration of MMP-7 in the stool is linked to a decreased serum MMP-7 concentration. MMP-7 showed a mediation effect on the association between inflammation and liver fibrosis in BA patients. Serum MMP-7 was the only significant predictor at six weeks post-KPE and the most accurate predictor at three months post-KPE of survival with the native liver (SNL) in two years.

\section{Conclusion:}

As one of the critical factors associated with BA occurrence and progression, serum MMP-7 can be used for early diagnosis of BA and post-KPE MMP-7 level is the earliest prognostic biomarker so far.

\section{Introduction}

Biliary atresia (BA) is one of the most severe cholestatic liver diseases in infants and the most frequent indication for paediatric liver transplantation (LTx) globally. (1-3) KPE could establish biliary-enteric drainage and offer the potential for long-term survival or postponed LTx in patients with BA. Early diagnosis and timely Kasai portoenterostomy (KPE) are correlated with favourable prognoses.(4-7) Unfortunately, similar clinical manifestations and overlapping examination results make an early and prompt diagnosis of BA extremely challenging. $(8,9)$ In addition, the cost-effectiveness of BA screening is still debatable, and the required infrastructure for screening is currently unavailable in most countries and regions.(10) Several studies have confirmed the diagnostic value of serum matrix metalloproteinase-7 (MMP-7) for BA and recovered the association between serum MMP-7 and bile duct epithelial injury and liver fibrosis.(11-14) A previous research reported that BA neonates have lower serum MMP-7 levels; thus, more evidence is needed to prove serum MMP-7's diagnostic value in BA neonates.(13) 
Failed KPE is common in BA patients, and the vast majority of liver transplants occur in BA children less than two years of age.(3) A Markov model simulation found an increase of 17.45 additional expected life years when a LTx was performed in a patient with a Paediatric End Stage Liver Disease (PELD) score of 15-25 with one or fewer systemic complications as compared to those with PELD scores $>25$ who had more than one systemic complication.(15) Identifying early predictors of progressive liver fibrosis would help surgeons plan individualized treatment interventions and determine the appropriate time for performing LTX. Clearance of jaundice at three months post-KPE is widely used in the evaluation of the prognosis of BA patients.(16) Therefore, this time point was chosen to evaluate the capacity of each prognostic factor in the following studies. Few studies reported the prediction value of dynamic patterns of serum biomarker levels in post-KPE BA infants for LTX.

In this multicentre prospective study, we evaluated the role of serum MMP-7 in diagnosing BA in cholestatic neonates. We also investigated the utility of dynamic patterns of serum MMP-7 for predicting two-year survival with native liver (SNL) in post-KPE BA patients.

\section{Materials And Methods}

\subsection{Study design and population}

From August 2017 to February 2020, a total of 5 hospitals participated in this prospective study (Department of Paediatric Surgery, Union Hospital, Tongji Medical College, Huazhong University of Science and Technology, Wuhan; Department of Neonatal Surgery, Xi'an Children's Hospital, Xi'an; Department of Paediatric Surgery, Wuhan Children's Hospital, Tongji Medical College, Huazhong University of Science and Technology, Wuhan; Department of General Surgery, Shenzhen Children's Hospital, Shenzhen; Department of General Surgery, Hebei Children's Hospital). A total of 440 cholestasis patients (direct bilirubin level of $>17 \mu \mathrm{mol} / \mathrm{L}$ ) with unclear diagnoses were included in this study.(17) Exclusion criteria included refusal to perform the MMP-7 test and known diagnosis/cause of cholestasis before the test. All patients underwent a workup for cholestasis, including general information, blood tests, urine tests, abdominal sonograms, and metabolic or genetic workups, and all serum MMP-7 level tests were performed in Wuhan Union Hospital. The diagnosis of BA was confirmed by intraoperative cholangiography, HSST signs(18) and pathological pictures. The final diagnosis for each included patient was made by paediatric hepatologists and paediatric surgeons together. The diagnostic criteria for BA were the same as those noted in a previous study.11 (Figure 1)

Follow-up of BA patients was performed at one week, two weeks, one month, six weeks, two months, three months, six months and then every six months post-KPE. Serum MMP-7 levels and liver function tests were detected in BA patients at each follow-up visit. All stool samples of BA patients were collected before discharge (usually 2 weeks post-KPE) and stool MMP-7 levels were tested. The follow-up endpoint was the date of LTX or death. SNL was defined as normal bilirubin levels and no cholestatic complications at the second year visit post-KPE. Informed and written consent to participate in the study was obtained from all patients' parents. 


\subsection{MMP-7 sample acquisition and measurement}

There were three ways to obtain MMP-7 serum samples in the current study: (1) No transportation required (blood sample from the Wuhan Union Hospital): Blood samples from 224 subjects were collected in a coagulation-promoting tube and placed in a $4^{\circ} \mathrm{C}$ refrigerator. (2) Transportation time less than 12 hours (other two hospitals in Wuhan): the blood samples from 83 subjects were gathered, stored in an ice box $\left(\leq 4^{\circ} \mathrm{C}\right)$ and transported to Wuhan Union Hospital for tests. For the above two situations, the blood samples were tested directly after centrifugation $(4000 \mathrm{rpm} / 5 \mathrm{~min})$ at $4^{\circ} \mathrm{C}$. (3) Transportation time greater than 12 hours: serum samples from 133 subjects were packed in a thermally isolated box filled with dry ice and transported to Wuhan Union Hospital. Samples were tested directly after they were received.

According to the protocol, serum and stool MMP-7 levels were measured using a Human Total MMP-7 Quantikine ELISA Kit (R\&D, USA). The dilution times were 8 and 32 for serum samples. Stool samples were washed with PBS three times immediately after collection. The final concentration of stool was $1 \mathrm{~g}$ to $9 \mathrm{ml} \mathrm{PBS}$, and the sample was subjected to ultrasound for 30 seconds. The stool solution was centrifuged for 5 minutes at $5000 \mathrm{~g}$, and the supernatant was used for ELISA tests.

\subsection{Simulation experiments of MMP-7 degradation}

Whole blood or serum samples were stored at room temperature, $4^{\circ} \mathrm{C}$, and dry ice, respectively. MMP-7 levels were detected at different time points: half an hour, 1 hour, 2 hours, 3 hours, and 4 hours for room temperature (RT); 1 hour, 2 hours, 4 hours, 8 hours, 12 hours, 24 hours, 36 hours, 48 hours, and 72 hours for $4^{\circ} \mathrm{C}$; half a day, 1 day, 2 days, 3 days, and 4 days for dry ice $(n=9)$. The freezing and thawing procedures were performed 1-5 times to explore the effect of freeze-thaw on MMP-7 degradation.

\subsection{MMP-7 expression and gene polymorphism}

All exons and promoter regions of MMP-7 in 7 patients with low serum MMP-7 levels (LSM, $<26.73$ $\mathrm{ng} / \mathrm{ml}$ ) and 33 patients (randomly chosen from 207 BA patients with high serum MMP-7 levels) with high serum MMP-7 levels (HSM, >26.73 ng/m) were sequenced using the Sanger method.

\subsection{Inflammation grades and fibrosis classifications}

The liver pathology of 256 patients $(B A=191$; non-BA $=65$, laparoscopic biliary lavage) undergoing surgery was analysed and recorded independently by two pathologists, and discrepancies were resolved by discussion. The stages of inflammation and fibrosis classifications were defined according to the Scheuer scale system, ranging from $\mathrm{G} 0-4$ and $\mathrm{S} 0-4 .(12)$

\subsection{Statistical analysis}

Categorical variables are described as frequencies and percentages; continuous variables are described as the mean (standard deviation) or median (interquartile range) according to the distribution of the data. 
The diagnostic cut-off value of serum MMP-7 was determined by receiver operating characteristic (ROC) analyses and area under the curve (AUC). Spearman's correlation was used to analyse the correlation between MMP-7 and different factors. The stepwise multivariable linear regression model was built considering all significant variables from the univariate regression. Mediation effect of MMP-7 on the association between inflammation and liver fibrosis were tested by Bootstrap proposed by Preacher \& Hayes.(19) Kaplan-Meier survival curves and log-rank tests were used to determine the ability of different factors to predict prognoses. Significant differences between two groups were tested by Student's t test or the Mann-Whitney $U$ test. The data were analysed using SPSS 26.0, and $p<0.05$ was used to determine significance.

\subsection{Ethics approval}

The research programme was approved by the Institutional Review Board of each center, and this clinical trial was approved and registered in the Chinese clinical trial registry (Number: ChiCTR1900028456). The medical records of all included patients were collected and analysed.

\section{Results}

\subsection{Demographic characteristics}

Of the 440 patients, 214 were diagnosed with BA, and 226 non-BA jaundice patients were recognized as the control group. All included patients were followed up to confirmation of diagnoses, and the diagnoses of 440 patients were listed in Supplementary Table 1. The baseline demographic, clinical, and laboratory profiles of the included patients were shown in Table 1.

\subsection{Optimal threshold value and age-related changes in serum MMP-7 levels}

In neonate cholestasis patients $(\mathrm{n}=65 ; \mathrm{BA}=34$, non-BA $=31$ ), using a cut-off value of $>26.73 \mathrm{ng} / \mathrm{ml}$, serum MMP-7 had a sensitivity $=91.2 \%$, specificity $=100 \%$, and AUC of 0.954 [95\% Cl: $0.897-1.000]$. (Supplementary Figure 1) In order to reduce missed opportunities for early BA diagnosis in cholestasis patients, we examined the diagnostic accuracy of using $>26.73 \mathrm{ng} / \mathrm{mL}$ in all patients without age limit (n $=440 ; \mathrm{BA}=214$, non-BA $=226$ ). Using a cut-off value of $>26.73 \mathrm{ng} / \mathrm{mL}$ in all cholestasis patients, serum MMP-7 had a sensitivity $=96.7 \%$, specificity $=95.6 \%$, and AUC of 0.983 [95\%Cl: $0.970-0.996]$. The positive predictive value (PPV), negative predictive value (NPV), and diagnostic efficiency of serum MMP-7 level $>26.73 \mathrm{ng} / \mathrm{mL}$ for predicting BA were $95.39 \%, 96.86 \%$, and $96.14 \%$, respectively. (Supplementary figure 2)

To investigate whether MMP-7 was age-related, we divided the included patients into three age groups (Group A: $4-28$ days, $n=65$, Group B: $29-60$ days, $n=234$, Group $C: \geq 61$ days, $n=141$ ). Analysis based on age groups showed that BA patients in group $C$ had significantly higher serum MMP-7 levels than those in group $A$ and group B. No significant differences were identified between group A and group B. In non-BA patients, no age-related differences were noted (Supplementary figure 3).

\subsection{Reasons for low serum MMP-7 level in BA patients}


Although serum MMP-7 has high diagnostic accuracy, 7 (3.27\%) BA patients in our cohort had serum MMP-7 lower than $26.73 \mathrm{ng} / \mathrm{ml}$. To assess the reason for the low MMP-7 expression level in these BA patients, we performed polymorphism sequencing on exons and promoter regions of MMP-7. The heterozygote mutation percentage of $\mathrm{c} .410 \mathrm{G}>\mathrm{A}(\mathrm{rs} 17884789 \mathrm{C}>\mathrm{T})$ in exon 3 was significantly increased in LSM patients compared with HSM patients $(30.00 \%$ vs $3.03 \%, P=0.032)$. (Supplementary Table 2)

As a small molecular weight protein, MMP-7 showed relatively poor stability in vitro. Since all serum samples were centralized to one center for testing, a standard transportation process should be developed.

How different transportation environments impacted serum MMP-7 levels were simulated to reduce the influence of external factors during transportation. MMP-7 degradation rates under different conditions (room temperature, $4^{\circ} \mathrm{C}$, and dry ice) are presented in Supplementary Figure 4. The level of MMP-7 was stable in both whole blood and serum on dry ice. In the $4^{\circ} \mathrm{C}$ refrigerators, serum MMP-7 protein levels were reduced to less than $90 \%$ after $12 \mathrm{~h}$ (whole blood) and $48 \mathrm{~h}$ (serum). At room temperature (RT), whole blood and serum MMP-7 degradation exceeded $50 \%$ after 4 hours (Supplementary Figure 4A). Each freezing-thawing procedure resulted in an approximately 15\% reduction in MMP-7 serum levels (Supplementary Figure 4B).

\subsection{Correlation of serum MMP-7 levels with multiple clinical characteristics at diagnosis}

A total of 191 of the 214 BA patients underwent surgery, and liver biopsies were obtained. Ten patients with histological cirrhosis and decompensation were referred to other departments for liver transplantation, and 13 patients did not have consent for KPE. Hence, a total of 191 cases were included for the following correlation analysis. Serum MMP-7 levels at diagnosis were found to positively correlate with total bilirubin (TBIL), direct bilirubin (DBIL), alanine aminotransferase (ALT), aspartate aminotransferase (AST), $\mathrm{Y}$-glutamyl transpeptidase ( $\mathrm{Y}-\mathrm{GT}$ ) at diagnosis and fibrosis classification and inflammation grade at KPE in BA patients (Supplementary Table 3). The multivariable linear regression model examining serum MMP-7 levels is summarized in Supplementary Table 4. The $\mathrm{Y}$-GT level at diagnosis and inflammation grades and fibrosis classifications at KPE were significant factors associated with serum MMP-7 levels at diagnosis. Moreover, MMP-7 showed a significant mediation effect on the association between inflammation and liver fibrosis in BA infants; however, no mediation effect was identified in non-BA infants (Table 2). The result indicated that MMP-7 could be a critical factor associated with inflammation-induced fibrogenesis in BA.

\subsection{Dynamic changes in serum MMP-7 in BA patients post-KPE}

Eighty-three BA patients who had a follow-up of less than two-year or were lost to follow-up after discharge were therefore excluded from the dynamic trend analysis. No significant differences were identified between the demographics of the included and excluded patients and SNL percentages of each centre (Supplementary table 5, 6). Hence, 108 BA patients were included for the following analysis. 62 (57.41\%) and 51 (47.22\%) post-KPE BA patients had event-free survival with their native liver (SNL) during 
the first and second-year post-KPE, respectively. Four patterns of changes in serum MMP-7 levels postKPE were observed. Detailed dynamic post-KPE serum MMP-7 changes in four representative patients are shown in Figure 2. MMP-7 pattern one $(n=11,10.19 \%)$ involved a rapid decline and then gradually increased or remained relatively stable at a low level (below the postoperative level), finally presenting a downward trend (Figure 2A). MMP-7 pattern two $(n=29,26.85 \%)$ showed elevated or fluctuating MMP-7 levels around a relatively high level and then a stable downward trend (Figure 2B). MMP-7 pattern three ( $\mathrm{n}$ $=51,47.22 \%$ involved a drastic fluctuation in the first 1-2 months post-KPE and subsequently elevated MMP-7 levels, even though the jaundice-free status ever achieved post-KPE (Figure 2C). MMP-7 pattern four ( $n=17,15.74 \%$ ) involved a continuously rising serum MMP-7 level post-KPE and usually had persistent jaundice (Figure 2D). The percentage of SNL patients at the two-years visit in the dynamic pattern one to four is $100 \%, 79.31 \%, 31.37 \%$, and $5.88 \%$, respectively. The dynamic trends of serum MMP7 levels in BA patients post-KPE with different prognoses (SNL vs. non-SNL) are summarized in Figure 2E. Significant differences between the two groups began to appear from four weeks post-KPE.

To determine the reason for the rapid decline in serum MMP-7 levels (pattern one) in some BA patients post-KPE, we further analysed MMP-7 levels in stool samples of 40 post-KPE BA patients. In response to the two changing directions (increase/decrease) in serum MMP-7 levels, two stool MMP-7 level dynamic patterns were observed. The detailed post-KPE serum and stool MMP-7 levels of two representative patients are shown in Figure 2 F, G. Patient 1 had a rapidly increased stool MMP-7 level with a decreasing/fluctuating serum MMP-7 level (Stool pattern 1, $n=17$, Figure 2F). Patient 2 had a stable low stool MMP-7 level; however, her serum MMP-7 level increased continuously after KPE (stool pattern 2, n = 3, Figure 2G). The stool MMP-7 levels were almost undetectable in BA patients at diagnosis. SNL patients had significantly higher stool MMP-7 levels post-KPE than LTx patients. (Supplementary Table 7)

\subsection{Correlation of serum MMP-7 levels with multiple clinical characteristics at 3 months post- KPE}

Supplementary Table 8 shows the positive correlations between postoperative clinical indicators and serum MMP-7 levels at three months post-KPE. The multivariable linear regression model showed that total bilirubin, Fibro Touch results and APRI were significantly associated with serum MMP-7 levels at three months post-KPE (Supplementary Table 9).

\subsection{The power of postoperative clinical indicators to predict SNL at two years post-KPE}

The average MMP-7 levels at diagnosis were not different between BA patients who survived with native liver (SNL) or not at two years post-KPE (68.95, IQR: $55.73-106.26 \mathrm{ng} / \mathrm{ml}$; vs. 74.43, IQR: 58.57 - 96.13 $\mathrm{ng} / \mathrm{ml}, \mathrm{p}=0.808)$. Age was the only preoperative indicator identified to have predictive power for SNL at two years post-KPE (SNL: $43.96 \pm 2.93$ days vs. non-SNL: $57.49 \pm 3.79$ days, $p=0.007$ ). Serum MMP-7 was the only indicator with significant differences at six weeks post-KPE, while all listed postoperative indicators at three months post-KPE showed significant differences (Supplementary Table 10). The ROC analyses showed varying degrees of power for serum MMP-7 levels, total bilirubin, direct bilirubin, $\mathrm{Y}-\mathrm{GT}$, Fibro Touch levels, and APRI post-KPE in predicting SNL at two years post-KPE (Figure 3 ) and MMP-7 at three months post-KPE having the highest AUC (0.8612, 95\% Cl: 0.790-0.932). Delong's test showed that 
serum MMP-7 level at six weeks and three months post-KPE has the highest prognostic value among all current minimally invasive indicators (Supplementary Table 11). However, the prediction ability of serum MMP-7 did not increase when combined with other indicators at six weeks post-KPE.

We also employed Kaplan-Meier survival analysis and Cox regression to ascertain which factors had strong correlations with SNL at two years post-KPE in BA patients. Figure 4 illustrates survival curves with log-rank tests for MMP-7 > $75.22 \mathrm{ng} / \mathrm{ml}$, TBIL > $35.6 \mu \mathrm{mol} / \mathrm{L}, \mathrm{DBIL}>18.9 \mu \mathrm{mol} / \mathrm{L}, \mathrm{GGT}>306.1 \mathrm{U} / \mathrm{L}$, APRI > 2.5448 , and Fibro Touch $>19.35$ at 3 months post-KPE. The Cox regression results showed that serum MMP-7 level, total bilirubin, and APRI at 3 months after KPE were significant indicators of SNL at two years post-KPE and that serum MMP-7 level had the highest hazard ratio $(5.34,95 \% \mathrm{Cl}$ [2.50 - 11.39]) (Supplementary Table 12).

\section{Discussion}

Several studies proved the diagnostic value of serum MMP-7 levels in BA patients. $(11,13,14)$ The inconsistency of the cut-off value of serum MMP-7 among different centres could be attributed to the different ELISA kits, differences between laboratories, different disease distributions in the control group, and different average ages of the included patients. The results of this study showed that serum MMP-7 could be applied in neonate BA diagnosis. The high sensitivity and specificity were maintained when applying the optimal cut-off value in neonate cholestasis patients to all patients for BA diagnosis. To find more BA patients at an early stage, we recommend using $26.73 \mathrm{ng} / \mathrm{ml}$ for BA diagnosis.

Our previous retrospective study and this prospective study showed that serum MMP-7 levels were lower than diagnostic cut-off values in a small number of BA patients.(14) This study provided two possible reasons. The first reason is that MMP-7 ( $r 17884789 \mathrm{C}>\mathrm{T}$ ) mutation may lead to low serum MMP-7 levels. A previous study focused on liver cirrhosis reported that MMP-7 (rs17884789 C > T) mutation could lead to different protein phenotypes, and the mutated protein is mainly distributed in the extracellular membranes and rarely secreted into the blood.(20) However, the MMP-7 (rs17884789 C > T) mutation was only identified in three of seven patients in the LSM group. Whether the low serum MMP-7 levels in another 7 cases were related to the polymorphism of other introns or exons needs further study. Almost no T alle exists at SNP rs17884789 except for the Asian population (1000 genomes project phase 3 allele frequencies from the ensemble), which indicated serum MMP-7 might have higher diagnostic accuracy in other races. The second reason is that MMP-7 decreased with increasing temperature, storage time, and freezing-thawing times. Long-term storage or transportation at $4^{\circ} \mathrm{C}$ or RT and repeated freeze-thaw events accelerate the degradation of MMP-7. Standardized specimen acquisition, transportation, and storage are essential for obtaining accurate results of serum MMP-7.

MMP-7 was a primary pathogenic mediator in the evolution of fibrotic lesions by degrading E-cadherin in kidney fibrosis.(21) In idiopathic pulmonary fibrosis and kidney fibrosis, MMP-7 levels in serum or urine correlate very well with clinical symptoms as well as prognosis. $(7,21,22)$ Different from other chronic liver fibrosis diseases, the inflammation-induced ductular reaction is the leading cause of liver fibrosis in 
cholangiopathies such as BA.(23) Chatmanee et al. found that preoperative serum MMP7 levels were correlated with inflammation classifications.(12) Jiang et al. reported that the preoperative serum MMP-7 level was correlated with the fibrosis stage in liver biopsies, whereas no significance was found within different inflammation grades.(11) In the current study, we found that serum MMP-7 level at diagnosis positively correlated with both inflammation and fibrosis grades. We also found a significant mediation effect of serum MMP-7 between inflammation and fibrosis in BA patients, which suggested that MMP-7 was produced by severe bile duct inflammation and promoted liver fibrosis through the E-cadherin/ $\beta$ catenin pathway.(24) Besides, serum MMP-7 at three months post-KPE was positively associated with indicators that affect the degree of liver fibrosis (total bilirubin levels, Fibro Touch results, and APRI). These results indicated that MMP-7 could be a factor that participates in the whole course of BA occurrence and development rather than the result of fibrosis.

Two previous studies reported that MMP-7 levels at six months post-KPE were positively correlated with the severity of liver fibrosis and native liver survival rates. $(13,25)$ To find more substantial evidence that can explain the mediation effect of MMP-7, we performed dynamic monitoring of serum and stool MMP7 levels in post-KPE BA patients and found that different dynamic patterns post-KPE serum and stool MMP-7 levels could lead to different surgical outcomes. A continuously high level of serum MMP-7 postKPE was more frequently seen in patients who failed KPE and needed LTx in two years. Moreover, a high stool MMP-7 level post-KPE is usually accompanied by a decrease in serum MMP-7. Thus, the observed decrease in serum MMP-7 levels post-KPE could be attributed to extrahepatic bile duct surgical reconstruction, which relieved biliary obstruction. A portion of MMP-7 could be excreted via the reconstructed bile duct. This finding indicated that the accumulation of MMP-7 in bile ducts, liver, or serum was related to progressive liver fibrosis and would lead to end-stage liver disease rapidly. While the massive excretion of MMP-7 through stool could maintain a low level of MMP-7 in the liver and bile duct, the fibrosis remains stable or even resolves over time in some post-KPE BA patients.

Prior studies have shown that TBIL, Y-GT, APRI, liver stiffness, fibrosis gene signature, interleukin-8, interleukin 12B, inflammation gene signature, and collagen hybridizing peptide levels could predict the prognoses of BA patients post-KPE.(26-33) In the current study, serum MMP-7 levels at six weeks and three months post-KPE made the most accurate predictions among all the indicators according to Delong's test of the ROC curves, and combination with other indicators would not increase the prediction accuracy. This finding highlighted the role of MMP-7 in the early prediction of two years SNL and suggested that MMP-7 is the forerunner of the known indicators related to BA progression. In the liver, MMP-7 is thought to be produced by various cells, such as glandular epithelial cells, cholangiocytes, and macrophages.(34-36) MMP-7 could reflect the early stage of bile duct injury before the bile duct obstruction develops. While other indicators (TBIL, DBIL, GGT, APRI, Fibro touch) could only reflect the result of bile duct obstruction and liver fibrosis. Moreover, simultaneous observation of serum and stool MMP-7 level could monitor the degree of inflammation, obstruction of the extrahepatic bile duct, and liver fibrosis more objectively than stool color. Similar to MMP-7's early diagnostic ability, this evidence suggested that MMP-7 could make better early predictions of SNL comparing to other prognostic factors. 
Most BA patients require liver transplants related to various morbid complications, and optimizing pretransplant care could maximize transplant outcomes.(3) As MMP-7 level measurement is a much less invasive detection method, dynamic observation of serum and stool MMP-7 levels post-KPE was recommended for every post-KPE BA patient. Further work is required to explore the metabolic pathway and pathogenesis of MMP-7 in the liver of BA patients.

Several limitations of the current study are worth noting. First, most included patients were from two provinces of China (Hubei and Shaanxi), potentially limiting the applicability of the study's results to other populations or ethnic groups. Second, the prospective study randomly included BA and non-BA patients who visited the outpatient department. Thus, there were some inconsistencies in the demographics of the two groups. However, no significant difference was identified in the average age between the two groups, given that serum MMP-7 levels are age-related in BA patients. Third, the heterogeneity of the sample storage, transportation, and multicentre nature of the study slightly decreases the credibility of the results but increases the study findings' external validity or generalizability. Finally, some patients were lost to follow-up, which may introduce some bias to the study.

To the best of our knowledge, the present study has been the first prospective, multicentre, large sample size study focused on the clinical application value of MMP-7 reported to date, providing a valuable basis for MMP-7's potential for cholestatic neonates differential diagnosis and an earliest prognostic biomarker in BA. Dynamic monitoring of serum and stool MMP-7 post-KPE is necessary for planning individualized treatment interventions and determine the appropriate time for performing LTX.

\section{Declarations}

Funding: National Natural Science Foundations of China (Grant Nos. 81670511 and 81270481 ).

Conflicts of interest: All authors declare no competing interests.

Ethics approval: The research programme was approved by the Institutional Review Board of each center, and this clinical trial was approved and registered in the Chinese clinical trial registry.

Consent to participate: Informed and written consent to participate in the study was obtained from all patients' parents.

Consent for publication: All authors agreed to publish this manuscript.

Availability of data and material: Reasonable request can obtain data from the corresponding author. STT has access to all data.

Code availability: Not applicable.

Authors' contributions: All authors contributed to the study conception and design. SQC and PPX collected, analysed the data, and drafted the manuscript; PY collected the data and provided analytical 
oversight; PPX, SQC, PY and STT designed and supervised the study; HBW, YQY, GQC, SL, YZ, XZ, XYL, HZN, LX and PCC revised the manuscript for important intellectual content; GQC and SL offered technical or material support; STT provided administrative support; all authors read and approved the final manuscript.

\section{References}

1. Bezerra JA, Wells RG, Mack CL, Karpen SJ, Hoofnagle JH, Doo E, Sokol RJ. Biliary Atresia: Clinical and Research Challenges for the Twenty-First Century. Hepatology. 2018;68:1163-73.

2. Feldman AG, Mack CL. Biliary Atresia: Clinical Lessons Learned. J Pediatr Gastroenterol Nutr. 2015;61:167-75.

3. Sundaram SS, Mack CL, Feldman AG, Sokol RJ. Biliary atresia: Indications and timing of liver transplantation and optimization of pretransplant care. Liver Transpl. 2017;23:96-109.

4. Lopez RN, Ooi CY, Krishnan U. Early and Peri-operative Prognostic Indicators in Infants Undergoing Hepatic Portoenterostomy for Biliary Atresia: a Review. Curr Gastroenterol Rep. 2017;19:16.

5. Obayashi J, Kawaguchi K, Manabe S, Nagae H, Wakisaka M, Koike J, Takagi M, et al. Prognostic factors indicating survival with native liver after Kasai procedure for biliary atresia. Pediatr Surg Int. 2017;33:1047-52.

6. Song Z, Dong R, Shen Z, Chen G, Yang Y, Zheng S. Surgical outcome and etiologic heterogeneity of infants with biliary atresia who received Kasai operation less than 60 days after birth: A retrospective study. Med (Baltim). 2017;96:e7267.

7. Tyraskis A, Davenport M. Steroids after the Kasai procedure for biliary atresia: the effect of age at Kasai portoenterostomy. Pediatr Surg Int. 2016;32:193-200.

8. Hartley JL, Davenport M, Kelly DA. Biliary atresia. Lancet. 2009;374:1704-13.

9. Sokol RJ, Shepherd RW, Superina R, Bezerra JA, Robuck P, Hoofnagle JH. Screening and outcomes in biliary atresia: summary of a National Institutes of Health workshop. Hepatology. 2007;46:566-81.

10. Harpavat S, Garcia-Prats JA, Anaya C, Brandt ML, Lupo PJ, Finegold MJ, Obuobi A, et al. Diagnostic Yield of Newborn Screening for Biliary Atresia Using Direct or Conjugated Bilirubin Measurements. Jama. 2020;323:1141-50.

11. Jiang J, Wang J, Shen Z, Lu X, Chen G, Huang Y, Dong R, et al. Serum MMP-7 in the Diagnosis of Biliary Atresia. Pediatrics 2019;144.

12. Lertudomphonwanit C, Mourya R, Fei L, Zhang Y, Gutta S, Yang L, Bove KE, et al. Large-scale proteomics identifies MMP-7 as a sentinel of epithelial injury and of biliary atresia. Sci Transl Med 2017;9.

13. Wu JF, Jeng YM, Chen HL, Ni YH, Hsu HY, Chang MH. Quantification of Serum Matrix Metallopeptide 7 Levels May Assist in the Diagnosis and Predict the Outcome for Patients with Biliary Atresia. J Pediatr. 2019;208:30-7.e31. 
14. Yang L, Zhou Y, Xu PP, Mourya R, Lei HY, Cao GQ, Xiong XL, et al. Diagnostic Accuracy of Serum Matrix Metalloproteinase-7 for Biliary Atresia. Hepatology. 2018;68:2069-77.

15. Arnon R, Leshno M, Annunziato R, Florman S, lyer K. What is the optimal timing of liver transplantation for children with biliary atresia? A Markov model simulation analysis. J Pediatr Gastroenterol Nutr. 2014;59:398-402.

16. Shneider BL, Brown MB, Haber B, Whitington PF, Schwarz K, Squires R, Bezerra J, et al. A multicenter study of the outcome of biliary atresia in the United States, 1997 to 2000. J Pediatr. 2006;148:46774.

17. Russo P, Magee JC, Boitnott J, Bove KE, Raghunathan T, Finegold M, Haas J, et al. Design and validation of the biliary atresia research consortium histologic assessment system for cholestasis in infancy. Clin Gastroenterol Hepatol. 2011;9:357-62.e352.

18. Zhou Y, Jiang M, Tang ST, Yang L, Zhang X, Yang DH, Xiong M, et al. Laparoscopic finding of a hepatic subcapsular spider-like telangiectasis sign in biliary atresia. World J Gastroenterol. 2017;23:7119-28.

19. Preacher KJ, Hayes AF. SPSS and SAS procedures for estimating indirect effects in simple mediation models. Behav Res Methods Instrum Comput. 2004;36:717-31.

20. Hung TM, Chang SC, Yu WH, Wang YW, Huang C, Lu SC, Lee PH, et al. A novel nonsynonymous variant of matrix metalloproteinase-7 confers risk of liver cirrhosis. Hepatology. 2009;50:1184-93.

21. Zhou D, Tian Y, Sun L, Zhou L, Xiao L, Tan RJ, Tian J, et al. Matrix Metalloproteinase-7 Is a Urinary Biomarker and Pathogenic Mediator of Kidney Fibrosis. J Am Soc Nephrol. 2017;28:598-611.

22. Song JW, Do KH, Jang SJ, Colby TV, Han S, Kim DS. Blood biomarkers MMP-7 and SP-A: predictors of outcome in idiopathic pulmonary fibrosis. Chest. 2013;143:1422-9.

23. Nomden M, Beljaars L, Verkade HJ, Hulscher JBF, Olinga P. Current Concepts of Biliary Atresia and Matrix Metalloproteinase-7: A Review of Literature. Front Med (Lausanne). 2020;7:617261.

24. Ke B, Fan C, Yang L, Fang X. Corrigendum. Matrix Metalloproteinase-7 and Kidney Fibrosis. Front Physiol. 2017;8:192.

25. Kerola A, Lampela H, Lohi J, Heikkilä P, Mutanen A, Hagström J, Tervahartiala T, et al. Increased MMP-7 expression in biliary epithelium and serum underpins native liver fibrosis after successful portoenterostomy in biliary atresia. J Pathol Clin Res. 2016;2:187-98.

26. Hukkinen M, Kerola A, Lohi J, Jahnukainen T, Heikkilä P, Pakarinen MP. Very low bilirubin after portoenterostomy improves survival of the native liver in patients with biliary atresia by deferring liver fibrogenesis. Surgery. 2019;165:843-50.

27. Jaramillo C, Guthery SL, Lowichik A, Stoddard G, Kim T, Li Y, Jensen MK. Quantitative Liver Fibrosis Using Collagen Hybridizing Peptide to Predict Native Liver Survival in Biliary Atresia: A Pilot Study. J Pediatr Gastroenterol Nutr. 2020;70:87-92.

28. Koga H, Wada M, Nakamura H, Miyano G, Okawada M, Lane GJ, Okazaki T, et al. Factors influencing jaundice-free survival with the native liver in post-portoenterostomy biliary atresia patients: results from a single institution. J Pediatr Surg. 2013;48:2368-72. 
29. Luo Z, Shivakumar P, Mourya R, Gutta S, Bezerra JA. Gene Expression Signatures Associated With Survival Times of Pediatric Patients With Biliary Atresia Identify Potential Therapeutic Agents. Gastroenterology. 2019;157:1138-52.e1114.

30. Pakarinen MP, Johansen LS, Svensson JF, Bjørnland K, Gatzinsky V, Stenström P, Koivusalo A, et al. Outcomes of biliary atresia in the Nordic countries - a multicenter study of 158 patients during 20052016. J Pediatr Surg. 2018;53:1509-15.

31. Shneider BL, Magee JC, Karpen SJ, Rand EB, Narkewicz MR, Bass LM, Schwarz K, et al. Total Serum Bilirubin within 3 Months of Hepatoportoenterostomy Predicts Short-Term Outcomes in Biliary Atresia. J Pediatr. 2016;170:211-7. :.e211-212..

32. Superina R, Magee JC, Brandt ML, Healey PJ, Tiao G, Ryckman F, Karrer FM, et al. The anatomic pattern of biliary atresia identified at time of Kasai hepatoportoenterostomy and early postoperative clearance of jaundice are significant predictors of transplant-free survival. Ann Surg. 2011;254:57785 .

33. Webb NL, Jiwane A, Ooi CY, Nightinghale S, Adams SE, Krishnan U. Clinical significance of liver histology on outcomes in biliary atresia. J Paediatr Child Health. 2017;53:252-6.

34. Fujishima S, Shiomi T, Yamashita S, Yogo Y, Nakano Y, Inoue T, Nakamura M, et al. Production and activation of matrix metalloproteinase 7 (matrilysin 1 ) in the lungs of patients with idiopathic pulmonary fibrosis. Arch Pathol Lab Med. 2010;134:1136-42.

35. Huang CC, Chuang JH, Chou MH, Wu CL, Chen CM, Wang CC, Chen YS, et al. Matrilysin (MMP-7) is a major matrix metalloproteinase upregulated in biliary atresia-associated liver fibrosis. Mod Pathol. 2005;18:941-50.

36. Saarialho-Kere UK, Crouch EC, Parks WC. Matrix metalloproteinase matrilysin is constitutively expressed in adult human exocrine epithelium. J Invest Dermatol. 1995;105:190-6.

\section{Tables}


Table 1

Demographics and laboratory findings of the study population

\begin{tabular}{|llll|}
\hline & BA $(\mathbf{n}=\mathbf{2 1 4})$ & Non-BA $(\mathbf{n}=\mathbf{2 2 6})$ & p \\
\hline Age, median (IQR), days & $50(35-66)$ & $50(35-65.25)$ & 0.975 \\
\hline $4-28$ & 34 & 31 & 0.587 \\
\hline $29-60$ & 113 & 121 & \\
\hline $61-189$ & 67 & 74 & $<0.001$ \\
\hline MMP-7, ng/ml & $61.26(47.87-102.04)$ & $12.57(8.22-18.19)$ & $<0.001$ \\
\hline Sex, $n(\%)$ & & & \\
\hline Male & $91(42.52)$ & $139(61.50)$ & $<0.001$ \\
\hline Female & $123(57.48)$ & $87(38.50)$ & $<0.001$ \\
\hline TB, $\mu$ mol/L & $180.85(137.40-224.58)$ & $133.30(94.45-185.80)$ & $<0.001$ \\
\hline DB, $\mu$ mol/L & $119.30(86.83-153.53)$ & $76.05(35.33-117.23)$ & $<0.001$ \\
\hline ALT, U/L & $110.00(62.75-193.50)$ & $59.00(29.00-120.00)$ & $<0.001$ \\
\hline AST, U/L & $171.00(104.00-280.00)$ & $108.00(55.00-172.00)$ & 0.022 \\
\hline Y-GT, U/L & $454.50(239.95-776.83)$ & $131.00(79.00-210.65)$ & $<$ \\
\hline PLT, G/L & $392.50(229.75-486.50)$ & $350.00(269.00-446.00)$ & 0.001 \\
\hline
\end{tabular}


Table 2

Mediation effect of MMP-7 on the association between inflammation and liver fibrosis in BA and Non-BA infants.

\begin{tabular}{|c|c|c|c|c|}
\hline \multicolumn{5}{|l|}{ BA } \\
\hline & coefficient & $\mathrm{p}$ & $\mathrm{LLCl}$ & ULCl \\
\hline constant & 1.7564 & $<0.001$ & 1.5110 & 2.0017 \\
\hline inflammation & 0.1354 & 0.0205 & 0.0211 & 0.2498 \\
\hline \multirow[t]{6}{*}{ MMP-7 } & 0.0037 & $<0.001$ & 0.0015 & 0.0058 \\
\hline & \multicolumn{4}{|c|}{ Direct effect of inflammation on fibrosis } \\
\hline & effect & $\mathrm{p}$ & $\mathrm{LLCl}$ & ULCl \\
\hline & 0.1354 & 0.0205 & 0.0211 & 0.2498 \\
\hline & \multicolumn{4}{|c|}{ Indirect effect of inflammation on fibrosis } \\
\hline & effect & & $\mathrm{LLCl}$ & $\mathrm{ULCl}$ \\
\hline MMP-7 & 0.1004 & & 0.0374 & 0.1688 \\
\hline \multicolumn{5}{|l|}{ Non-BA } \\
\hline & coefficient & $\mathrm{p}$ & LLCl & $\mathrm{ULCl}$ \\
\hline constant & 0.9096 & $<0.001$ & 0.4973 & 1.3218 \\
\hline inflammation & 0.3247 & 0.0358 & 0.0225 & 0.6270 \\
\hline \multirow[t]{6}{*}{ MMP-7 } & 0.0057 & 0.7058 & -0.0243 & 0.0357 \\
\hline & \multicolumn{4}{|c|}{ Direct effect of inflammation on fibrosis } \\
\hline & effect & $\mathrm{p}$ & $\mathrm{LLCl}$ & ULCl \\
\hline & 0.3247 & 0.0358 & 0.0225 & 0.6270 \\
\hline & \multicolumn{4}{|c|}{ Indirect effect of inflammation on fibrosis } \\
\hline & effect & & LLCl & ULCl \\
\hline MMP-7 & 0.0361 & & -0.1509 & 0.2418 \\
\hline
\end{tabular}




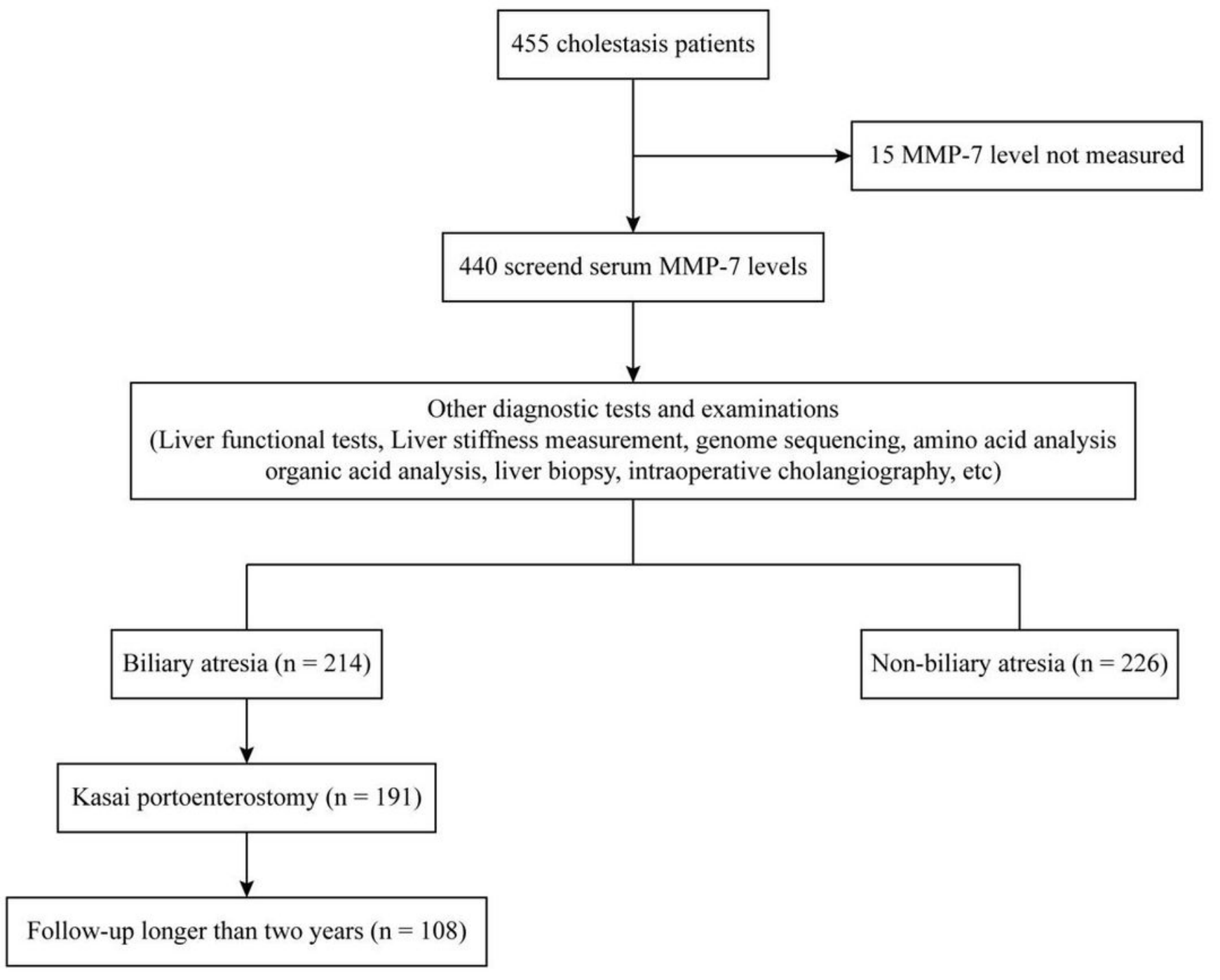

\section{Figure 1}

Flow chart of patient selection, diagnostic process, and follow-up 

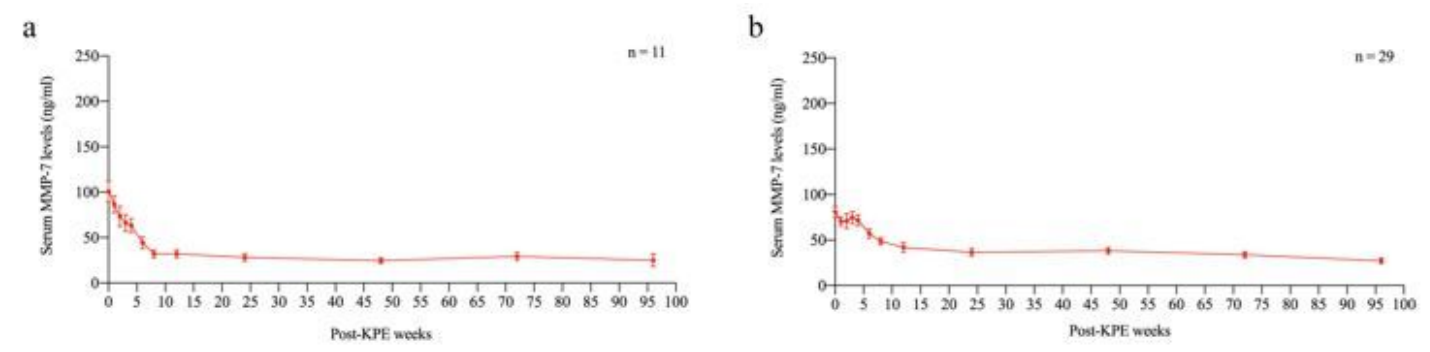

c

d
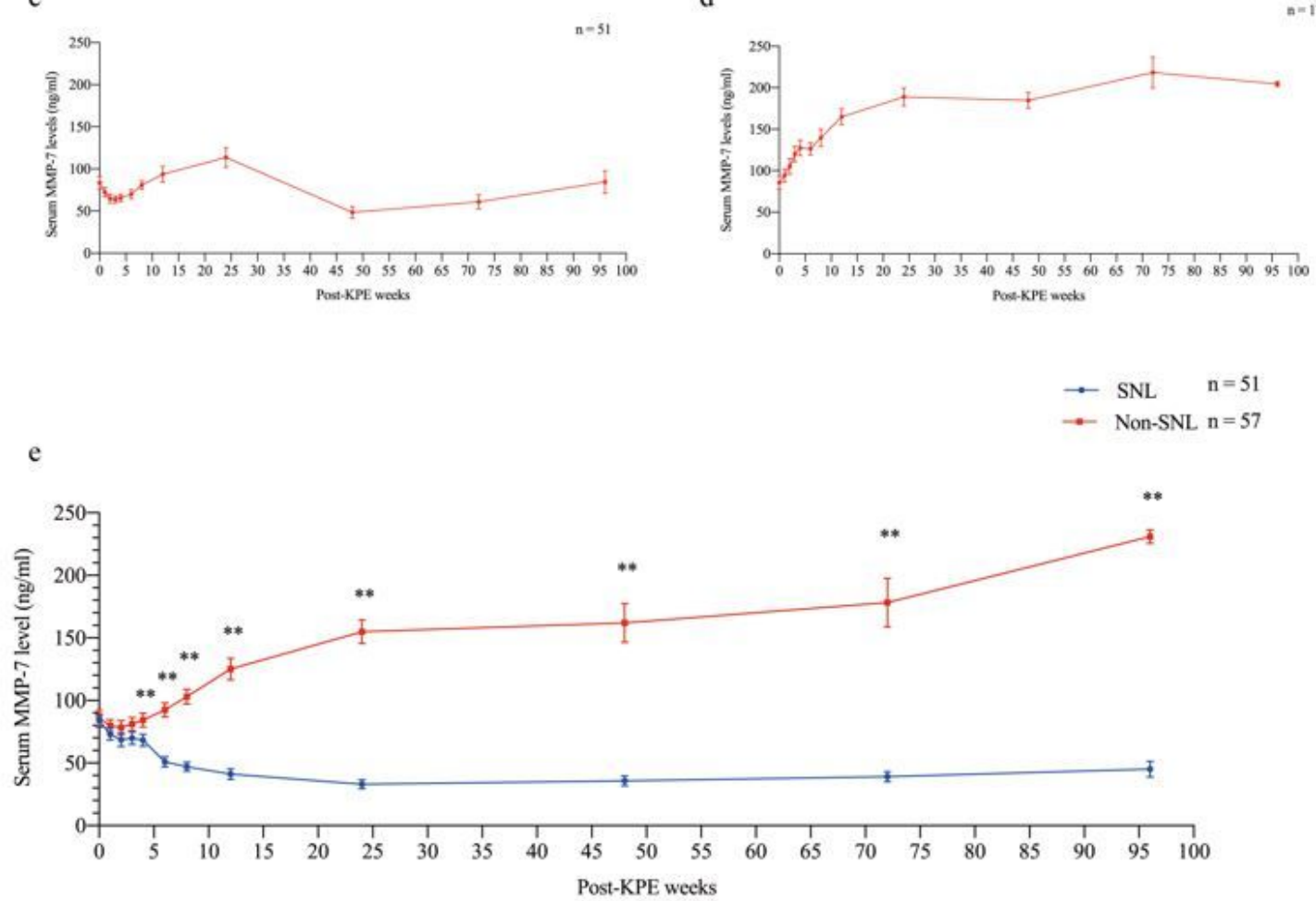

f

g
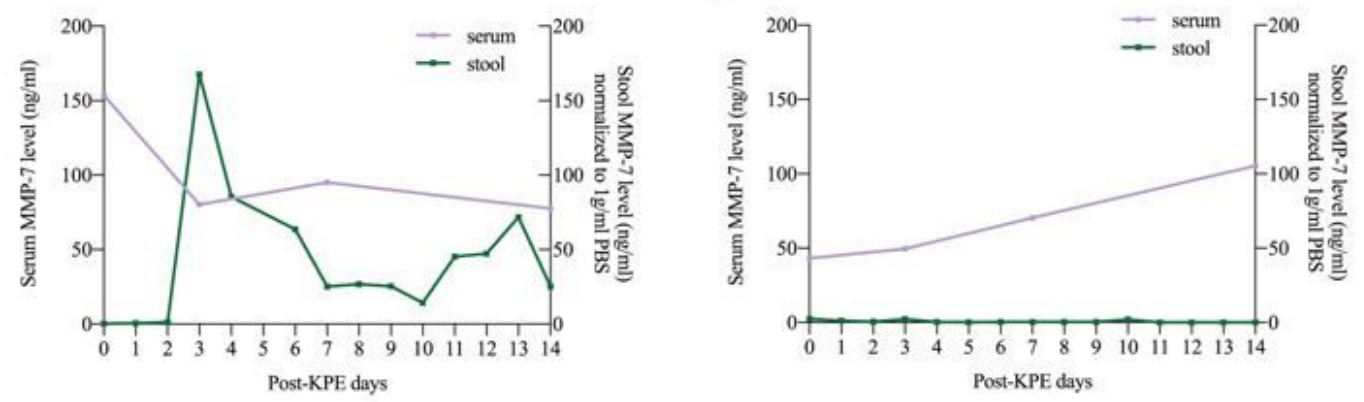

\section{Figure 2}

Dynamic MMP-7 patterns and comparison with total bilirubin levels. (a) pattern 1 (b) pattern 2 (c) pattern 3 (d) pattern 4. (e) Dynamic changes in serum MMP-7 levels after hepatoportoenterostomy in different prognosis groups. Dynamic patterns of stool MMP-7 levels $(\mathrm{f})$ pattern 1 (g) pattern 2 

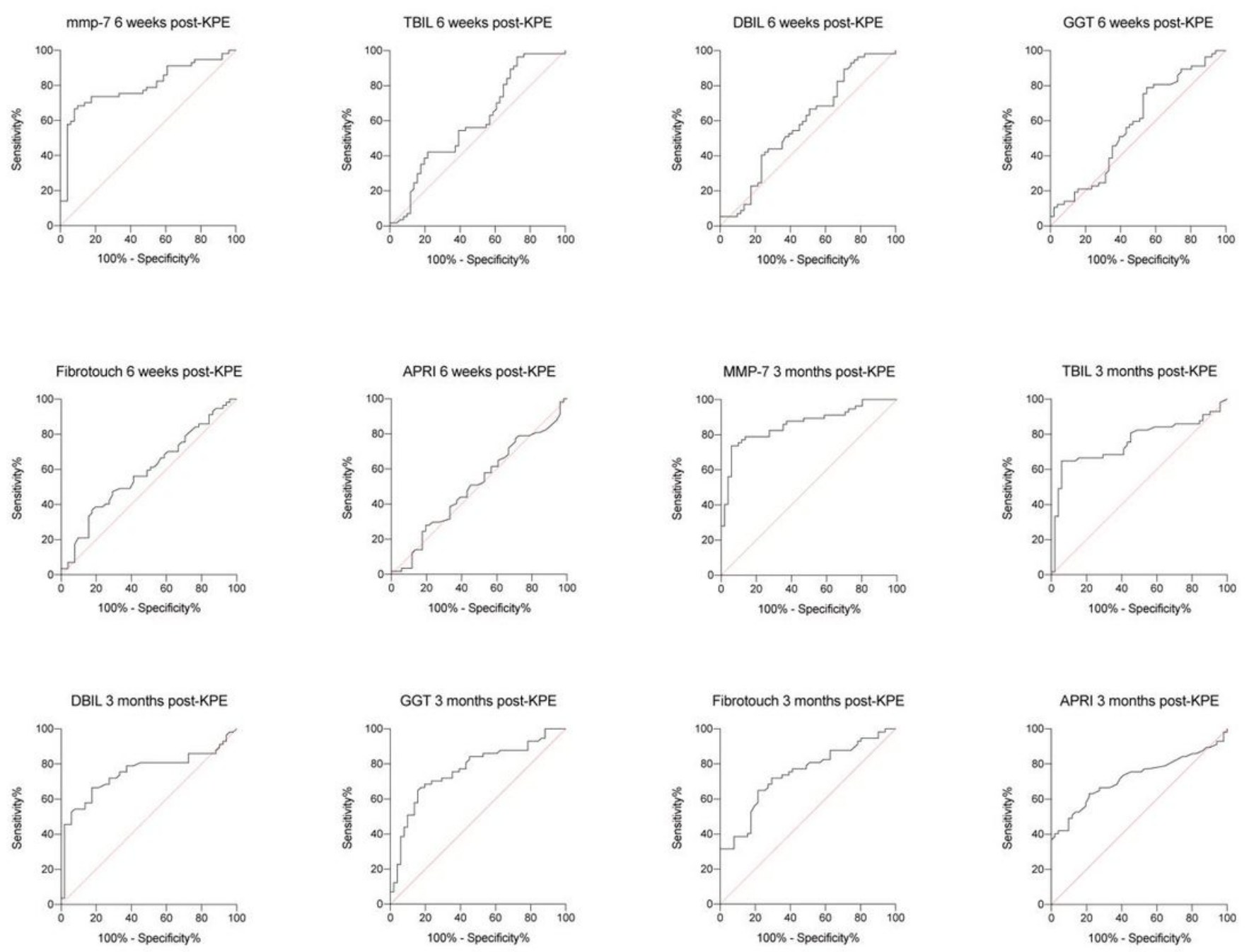

\section{Figure 3}

ROC curves of different post-KPE indicators at 6 and 3months post-KPE for prognosis prediction 


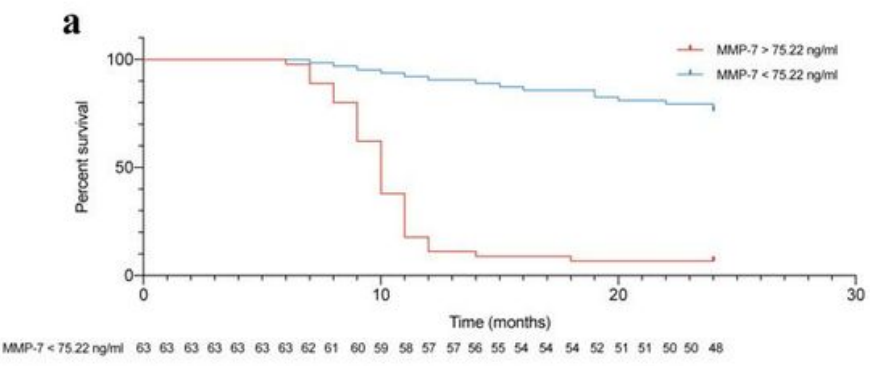

MMP.7 $>7522 \mathrm{mglml} 45 \quad 45 \quad 4545 \quad 45 \quad 454440 \begin{array}{llllllllllllllllll}36 & 28 & 17 & 8 & 5 & 5 & 4 & 4 & 4 & 4 & 3 & 3 & 3 & 3 & 3 & 3 & 3\end{array}$

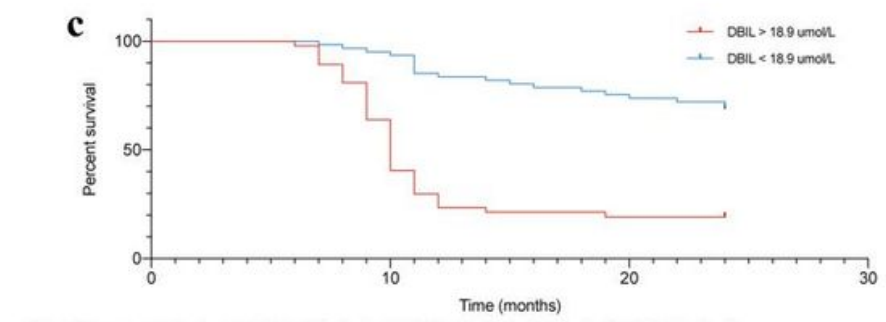

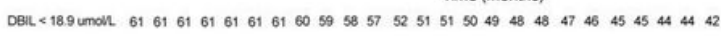

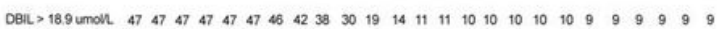

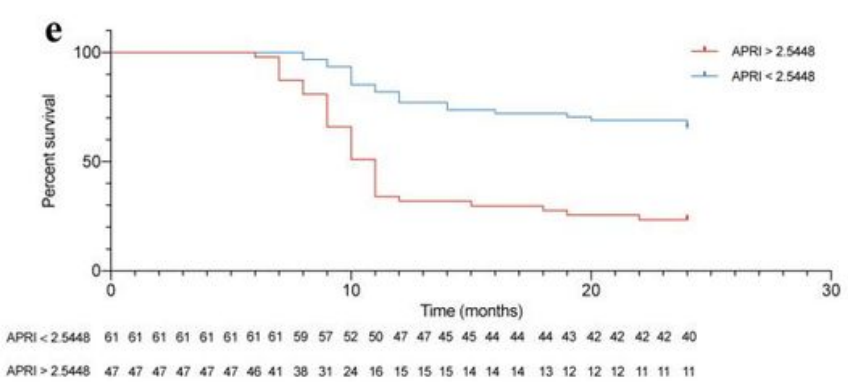

b

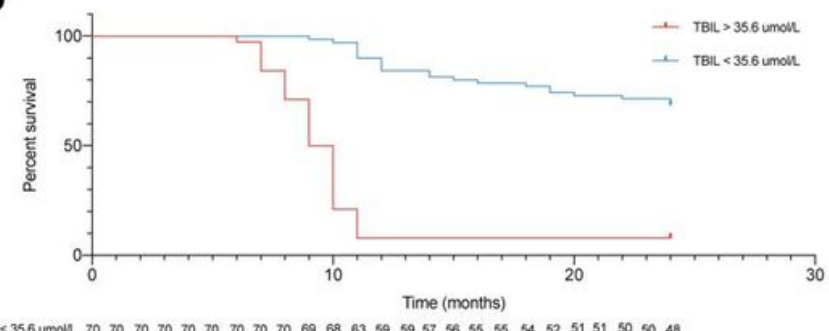

TBLL < 356 unmolL $7070707070707070706968635959575655 \quad 55 \quad 54525151 \quad 505048$

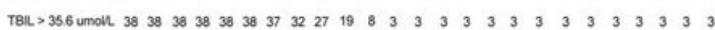

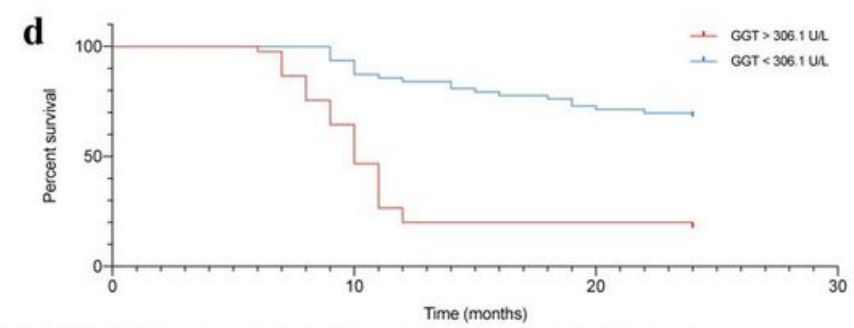

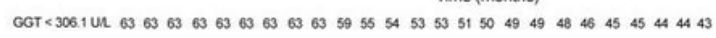

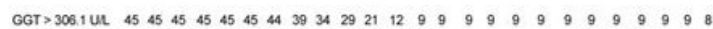

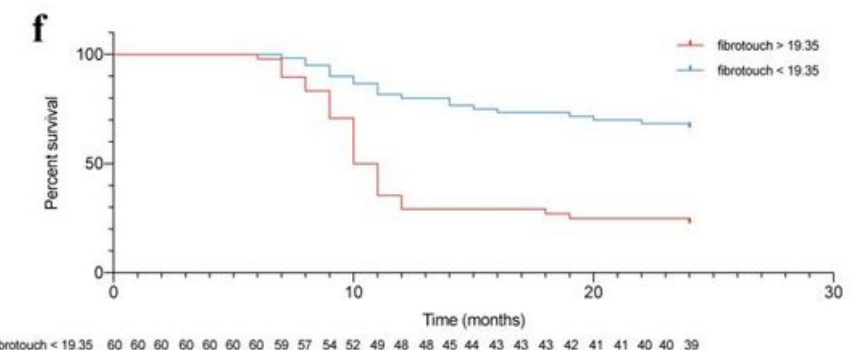

\section{Figure 4}

Kaplan-Meier survival curves of different post-KPE indicators at 3 months post-KPE for prognosis prediction (a) MMP-7 (b) TBIL (c) DBIL (d) y-GT (e) APRI (f) Fibro Touch

\section{Supplementary Files}

This is a list of supplementary files associated with this preprint. Click to download.

- SupplementaryTable1.docx

- SupplementaryTable11.docx

- SupplementaryTable2.docx

- SupplementaryTable6.docx

- SupplementaryTable7.docx

- Supplementarytable10.docx

- Supplementarytable3.docx 
- Supplementarytable4.docx

- Supplementarytable5.docx

- Supplementarytable12.docx

- Supplementarytable8.docx

- Supplementarytable9.docx

- supplementaryfigure1.tif

- supplementaryfigure2.tif

- supplementaryfigure3.tif

- supplementaryfigure4.tif 